\title{
Decreased expression levels of ELOVL6 indicate poor prognosis in hepatocellular carcinoma
}

\author{
HUI LI ${ }^{1}$, XIANLING WANG ${ }^{1}$, JUN TANG $^{2}$, HAIBO ZHAO $^{1}$ and MIN DUAN ${ }^{3}$ \\ ${ }^{1}$ Invasive Technology Department, Jining No. 1 People's Hospital, Jining, Shandong 272011; ${ }^{2}$ Invasive Technology \\ Department, Shandong Medical Imaging Research Institute, Jinan, Shandong 250021; ${ }^{3}$ Department of Physical Examination, \\ Jining First People's Hospital, Jining, Shandong 272000, P.R. China
}

Received November 18, 2018; Accepted June 13, 2019

DOI: $10.3892 / \mathrm{ol} .2019 .10974$

\begin{abstract}
The present study aimed to investigate the expression of elongation of very long-chain fatty acids family member 6 (ELOVL6) in hepatocellular carcinoma (HCC) tissues, and to determine its role in the development of HCC. A total of 377 HCC specimens were collected for tissue microarray and immunohistochemistry analyses. The ELOVL6 IHC score for HCC tissues was $0.97 \pm 0.71$, which was significantly lower than that of the matched adjacent normal tissues $(1.32 \pm 0.68 ; \mathrm{P}<0.001)$. Patients with low levels of ELOVL6 expression were older $(\mathrm{P}=0.014)$ and possessed larger sized tumors $(\mathrm{P}=0.039)$ than patients with high expression levels. Additionally, Kaplan-Meier analysis revealed that patients with low ELOVL6 expression levels also had significantly poorer overall $(\mathrm{P}<0.001)$ and disease-free $(\mathrm{P}=0.029)$ survival times, and a greater probability of recurrence. The tumor size, tumor-node-metastasis (TNM) stage, vascular invasion and ELOVL6 expression were all shown to be prognostic variables for overall survival in patients with HCC. Multivariate analysis revealed that vascular invasion $(\mathrm{P}<0.001)$, TNM stage $(\mathrm{P}<0.001)$ and ELOVL6 expression $(\mathrm{P}=0.001)$ were independent prognostic variables for overall survival. In addition, vascular invasion $(\mathrm{P}=0.032)$ and ELOVL6 expression $(\mathrm{P}=0.041)$ were independent risk factors for disease-free survival, and vascular invasion $(\mathrm{P}=0.019)$ and ELOVL6 expression $(\mathrm{P}=0.045)$ were independent risk factors associated with $\mathrm{HCC}$ recurrence. The present study revealed that in patients with HCC, ELOVL6 expression level was reduced in HCC tissues, and that higher ELOVL6 expression levels correlated with longer survival times. This indicates that ELOVL6 may serves as an independent marker of poor patient outcome.
\end{abstract}

Correspondence to: Dr Min Duan, Department of Physical Examination, Jining First People's Hospital, 269 Menci Avenue, Jining High-Tech Zone, Jining, Shandong 272000, P.R. China

E-mail: duanmin1978@163.com

Key words: elongation of very long-chain fatty acids family member 6, prognosis, hepatocellular carcinoma, biomarker

\section{Introduction}

Hepatocellular carcinoma (HCC) is the most common primary malignant tumor of the liver, with a mortality rate that ranks second globally $(1,2)$. Various etiologies of HCC have been reported, including chronic hepatitis virus infection, alcohol consumption and abnormal metabolism, and treatment is complex $(3,4)$. Although surgical resection may be curative under certain conditions, the majority of patients are diagnosed at a late stage when surgery is no longer effective (5). A specific biomarker for HCC has yet to be identified $(6,7)$, thus the early diagnosis of HCC and the discovery of predictive biomarkers have important clinical implications $(2,8,9)$. Investigating the pathogenesis of HCC by identifying abnormally expressed genes or proteins may considerably improve the diagnosis and treatment of the disease.

The association between abnormal lipid metabolism and tumorigenesis has attracted increasing attention (8-11). Tumor cells have altered proliferative and metabolic abilities compared with normal cells $(12,13)$, and the enzymes associated with fatty acid synthesis are upregulated in tumor tissues; this increases fatty acid synthesis and provides the necessary materials and energy to facilitate rapid tumor growth $(14,15)$. Of the associated enzymes, elongation of very long-chain fatty acids family member 6 (ELOVL6) is a highly conserved member of the endoplasmic reticulase family, which is involved in the formation of long-chain fatty acids $(16,17)$. Previous studies have found that the ELOVL6 gene serves an important role in the development and progression of breast cancer by regulating the metabolism of intracellular lipid components $(18,19)$. Further studies have revealed that HCC cells exhibit abnormal lipid metabolism $(20,21)$. Although the liver is the primary site of lipid metabolism $(22,23)$, there are currently no reports of the expression and significance of ELOVL6 in HCC, a cancer closely associated with metabolic disorders. ELOVL6 is a long chain fatty acid elongase, which may contribute to fatty acid storage. Until now, the study of ELOVL6 expression in $\mathrm{HCC}$ has remained limited. Hence, the present study aimed to investigate the expression levels of ELOVL6 in human HCC tissues, and to determine the relationship between the expression of ELOVL6 and the prognosis of patients with HCC. 


\section{Subjects and methods}

Subjects. A total of 377 paraffin-embedded HCC tissues were collected from the Jining No. 1 People's Hospital (Shandong, China) between January 2000 and July 2010. None of the patients received chemotherapy or radiotherapy prior to surgery. Patients received serological and imaging examinations to exclude recurrence or metastasis, and serum alpha-fetoprotein (AFP) level was determined. Abdomen ultrasonography, computed tomography and magnetic resonance imaging were utilized for physical examinations. Patients with missing data were excluded. The follow-up period was defined as the time interval between the date of surgery and that of death or the last follow-up. The mean follow-up interval was 30.89 \pm 28.18 months (range, 3.12-146.58 months); patient characteristics are outlined in Table I. The mean age of the patients with $48.94 \pm 12.78$ (range, $28-77$ years). The present study was approved by the Medical Ethics Committee of Jining No. 1 People's Hospital, and as a retrospective study, the requirement for informed patient consent was waived.

Tissue microarray (TMA) and immunohistochemistry (IHC). Following surgery, all specimens were immediately embedded in paraffin and stored at room temperature. Each tissue core (diameter, $0.6 \mathrm{~mm}$ ) was perforated and re-embedded from the labeled area using a tissue array (MiniCore) per the manufacturer's protocol. The expression level of ELVOL6A was detected in 377 tissue-pairs (cancerous and matched-noncancerous tissues); the specimens were fixed with $4 \%$ paraformaldehyde overnight at room temperature (RT), and subsequently processed using the biotin blocking Kit (Dark, Germany) at RT for $15 \mathrm{~min}$. The tissues were incubated with an anti-ELOVL6 antibody (1:1,000; cat. no. ab69857; Abcam) in a humid chamber at $4^{\circ} \mathrm{C}$ overnight. The tissues were washed 3 times with PBS and incubated with biotinylated goat anti-rabbit antibodies (1:200; cat. no. S0001; Affinity Biosciences) for $1 \mathrm{~h}$ at $37^{\circ} \mathrm{C}$. The sections were then stained with hematoxylin at room temperature for $10 \mathrm{~min}$, and observed under a light microscope (Olympus) at $\times 4$ and $\times 20$ magnification.

Semi-quantitative IHC was used to detect ELOVL6 protein expression levels according to the following intensity score criteria: 0 , negative staining; 1 , weak staining; 2 , moderate staining; and 3, strong staining. The final scores were calculated as a percentage of positive expression multiplied by the intensity score. The median IHC score was used as a cut-off to differentiate between high and low expression levels.

Oncomine database analysis. Oncomine ${ }^{\mathrm{TM}}$ (http://www. oncomine.org) is a web-based data-mining platform aimed to facilitate novel discoveries via genome-wide expression analysis $(24,25)$. The ELOVL6 gene was queried in the database and the results were filtered by selecting 'HCC' and 'Cancer' vs. 'Normal Analysis'. Comparisons between ELOVL6 mRNA expression levels in the HCC and adjacent normal tissues of 377 patients were analyzed using the Student's t-test.

Statistical analysis. Statistical analysis was performed using SPSS software (version 13; SPSS Inc.). The paired Student's t-test or $\chi^{2}$ test was used to assess the association between ELOVL6 expression level and clinicopathological variables. A survival curve was generated using the Kaplan-Meier method (log-rank test), and the multivariate Cox proportional hazards regression model was used to assess the independence of ELOVL6 as a predictive factor for HCC. $\mathrm{P}<0.05$ was considered to indicate a statistically significant difference.

\section{Results}

Expression of ELOVL6 in the HCC TMA. The HCC TMA $(\mathrm{n}=377)$ was used to determine ELOVL6 expression levels in HCC tissues. ELOVL6 was predominantly expressed in the cytoplasm of HCC cells. The ELOVL6 IHC score for HCC tissues was $0.97 \pm 0.71$, significantly lower than that of matched normal tissues $(1.32 \pm 0.68$; $\mathrm{P}<0.001$; Fig. 1). HCC data (Guichard Liver 2 Data) was also downloaded from the Oncomine Database (https://www.oncomine. org/resource/login.html) as validation data. These results also showed a reduction in ELOVL6 expression level in HCC tissues (supplementary Fig. 1).

Association between cytoplasmic ELOVL6 and HCC clinical features. To determine the clinical relevance of ELOVL6 expression in HCC, the association between ELOVL6 expression level and the clinical features of patients with HCC was evaluated. The median IHC score of the tumor tissues was 0.9 and a low level of ELOVL6 expression was observed in $67.9 \%(256 / 377)$ of the cases. The median age $(\mathrm{P}=0.014)$ and tumor size $(\mathrm{P}=0.039)$ were greater in patients with low levels of ELOVL6 expression, compared with those with high expression levels. The proportion of patients exhibiting vascular invasion was significantly higher in the low ELOVL6 expression group compared with patients in the high ELOVL6 expression group $(\mathrm{P}=0.005)$ (Table I).

Association between ELOVL6 expression level and the outcome of patients with HCC. To determine the prognostic value of ELOVL6 expression level in patients with HCC, Kaplan-Meier survival analysis was conducted using data from the 377 enrolled patients. Kaplan-Meier analysis revealed that patients with low ELOVL6 expression levels had significantly poorer overall survival times (Fig. 2A; $\mathrm{P}<0.001)$. Similarly, compared with patients with high ELOVL6 expression levels, disease-free survival time was shorter (Fig. 2B; $\mathrm{P}=0.029)$ and the probability of recurrence was higher (Fig. 2C; $\mathrm{P}=0.044$ ) in those with low ELOVL6 expression levels.

Univariate and multivariate analyses of prognostic variables in HCC. To evaluate whether ELOVL6 expression was an independent risk factor for patient outcome in $\mathrm{HCC}$, univariate and multivariate analyses were conducted. The tumor size, TNM stage, vascular invasion status and ELOVL6 expression were all shown to be prognostic variables for overall survival in patients with HCC. Multivariate analysis showed that only vascular invasion $(\mathrm{P}<0.001)$, TNM stage $(\mathrm{P}<0.001)$ and ELOVL6 expression $(\mathrm{P}=0.001)$ were independent prognostic variables for overall survival (Table II).

The risk factors associated with disease-free survival (Table III) and HCC recurrence were investigated further (Table IV). Univariate analysis revealed that age, TNM stage, 
Table I. Clinical variables in patients with hepatocellular carcinoma exhibiting low or high ELOVL6 expression levels.

\begin{tabular}{|c|c|c|c|}
\hline \multirow[b]{2}{*}{ Variable } & \multicolumn{2}{|c|}{ ELOVL6 expression level } & \multirow[b]{2}{*}{ P-value } \\
\hline & Low & High & \\
\hline Sample size & 256 & 121 & \\
\hline Age, years & & & 0.014 \\
\hline$>50$ & $134(52.3 \%)$ & $47(38.8 \%)$ & \\
\hline$\leq 50$ & $122(47.7 \%)$ & $74(61.2 \%)$ & \\
\hline Sex & & & 0.101 \\
\hline Male & $217(84.8 \%)$ & $110(90.9 \%)$ & \\
\hline Female & $39(15.2 \%)$ & $11(9.1 \%)$ & \\
\hline $\mathrm{AFP}, \mathrm{ng} / \mathrm{ml}$ & & & 0.069 \\
\hline$<20$ & $45(17.6 \%)$ & $31(25.6 \%)$ & \\
\hline$\geq 20$ & $211(82.4 \%)$ & $90(74.4 \%)$ & \\
\hline Cirrhosis & & & 0.670 \\
\hline Yes & $216(84.4 \%)$ & $100(82.6 \%)$ & \\
\hline No & $40(15.6 \%)$ & $21(17.4 \%)$ & \\
\hline Tumor size, $\mathrm{cm}$ & & & 0.039 \\
\hline$<5$ & $59(23.0 \%)$ & $40(33.1 \%)$ & \\
\hline$\geq 5$ & $197(77.0 \%)$ & $81(66.9 \%)$ & \\
\hline Differentiation & & & 0.347 \\
\hline Well-moderate & $20(7.8 \%)$ & $13(10.7 \%)$ & \\
\hline Poor-undifferentiated & $236(92.2 \%)$ & $108(89.3 \%)$ & \\
\hline TNM stage & & & 0.454 \\
\hline I-II & $112(43.8 \%)$ & $48(39.7 \%)$ & \\
\hline III-IV & $144(56.3 \%)$ & $73(60.3 \%)$ & \\
\hline Vascular invasion & & & 0.005 \\
\hline Yes & $53(20.7 \%)$ & $15(12.4 \%)$ & \\
\hline No & $203(79.3 \%)$ & $106(87.6 \%)$ & \\
\hline
\end{tabular}

ELOVL6, elongation of very long-chain fatty acids family member 6; AFP, alpha-fetoprotein; TNM, tumor-node-metastasis.

vascular invasion status and ELOVL6 expression were risk factors associated with disease-free survival. Multivariate analysis showed that vascular invasion $(\mathrm{P}=0.032)$ and ELOVL6 expression $(\mathrm{P}=0.041)$ were independent risk factors associated with disease-free survival. Only vascular invasion $(\mathrm{P}=0.019)$ and ELOVL6 expression $(\mathrm{P}=0.045)$ were independent risk factors for $\mathrm{HCC}$ recurrence.

Subgroup analyses of the prognostic value of cytoplasmic ELOVL6 expression in patients with HCC. Stratified survival analysis was conducted to reveal the prognostic implication of ELOVL6 expression in patients with HCC. Kaplan-Meier survival analysis illustrated that ELOVL6 expression was associated with overall survival in both the TNM stage I-II $(\mathrm{P}=0.048)$ and stage III-IV $(\mathrm{P}=0.003)$ groups in patients with tumor size $>5 \mathrm{~cm}(\mathrm{P}=0.014)$ and those with tumor size $\leq 5 \mathrm{~cm}$ $(\mathrm{P}=0.042)$. ELOVL6 expression was also associated with overall survival in younger $(\mathrm{P}=0.043)$ and older $(\mathrm{P}=0.014)$ patients with HCC (Fig. 3).

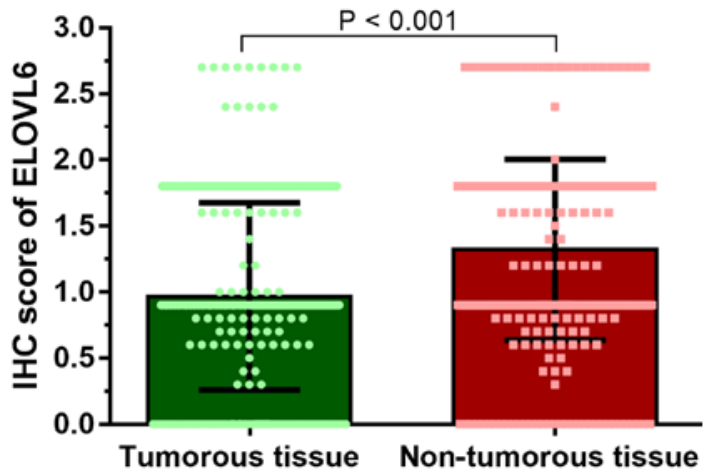

Figure 1. Expression level of ELOVL6 in HCC tissues. The ELOVL6 IHC score of $377 \mathrm{HCC}$ tumor tissues was $0.97 \pm 0.71$, significantly higher than that of matched normal tissues $(1.32 \pm 0.68 ; \mathrm{P}<0.001)$. ELOVL6, elongation of very long-chain fatty acids family member 6 ; HCC, hepatocellular carcinoma; IHC, immunohistochemistry.

\section{Discussion}

HCC is the most common malignant tumor of the liver (26-29). The result of ELOVL6 expression on the proliferation, invasion and metastasis of HCC cells was yet to be investigated, but was believed to provide insights into novel treatment options for patients with HCC. The present study confirmed that the expression level of ELOVL6 was decreased in HCC tissues, and that ELOVL6 expression was negatively associated with tumor size. Furthermore, a low expression level of ELOVL6 was associated with unfavorable outcome in patients with HCC. This indicates that ELOVL6 is a potential, novel therapeutic target and prognostic biomarker for HCC.

Fatty acids are essential components of biofilm lipids, signaling molecules and constituents of energy metabolism pathways (30-32). Among them, palmitic acid serves a prominent role in the formation of long-chain fatty acids containing 16 carbon atoms (C16:0), and studies have reported excessive accumulation of palmitic acid in breast cancer cells (33-35). ELOVL6 is a key enzyme in intracellular lipid metabolism, and has previously been associated with metabolism in fatty liver and diabetes (36). However, the relationship between metabolic reprogramming and expression of ELVOL6 in HCC has not previously been reported. In the present study, it was determined that tumor size was closely associated with ELOVL6 expression level. It is also possible that ELOVL6-associated lipid metabolism is able to promote tumor proliferation, though the specific mechanisms remain to be determined.

Moon et al (37) found that the conversion of palmitic acid to stearic acid (C18:0) was inhibited in ELOVL6 knock-out mice, suggesting that ELOVL6 is indispensable for palmitic acid metabolism (37). It has been speculated that ELOVL6 converts excess palmitic acid (C16:0), serving a role in tumor suppression. Kessler et al (38) found that in a mouse model of diethylnitrosamine-induced HCC, the expression level of ELVOL6 in cancerous tissues was lower than that in non-cancerous liver tissues. The present study was consistent with these results, where ELOVL6 expression level was also significantly reduced in HCC tissues. In addition, ELOVL6 expression was negatively associated with tumor size. 
Table II. Univariate and multivariate analyses of hepatocellular carcinoma patient variables for overall survival.

\begin{tabular}{|c|c|c|c|c|c|c|}
\hline \multirow[b]{2}{*}{ Variable } & \multicolumn{3}{|c|}{ Univariate analysis } & \multicolumn{3}{|c|}{ Multivariate analysis } \\
\hline & HR & $95 \% \mathrm{CI}$ & P-value & HR & $95 \% \mathrm{CI}$ & P-value \\
\hline Age, years & 0.992 & $0.983-1.001$ & 0.097 & & & \\
\hline Sex & 0.816 & $0.578-1.151$ & 0.247 & & & \\
\hline AFP & 1.097 & $0.835-1.442$ & 0.505 & & & \\
\hline Cirrhosis & 0.946 & $0.698-1.282$ & 0.721 & & & \\
\hline Tumor size, cm & 1.578 & $1.211-2.055$ & 0.001 & & & \\
\hline Differentiation & 1.472 & $0.984-2.204$ & 0.060 & & & \\
\hline TNM stage & 1.807 & $1.432-2.282$ & $<0.001$ & 1.591 & $1.244-2.036$ & $<0.001$ \\
\hline Vascular invasion & 3.266 & $2.463-4.331$ & $<0.001$ & 2.678 & $1.992-3.600$ & $<0.001$ \\
\hline ELOVL6 expression & 1.476 & $1.152-1.891$ & 0.002 & 1.509 & $1.174-1.939$ & 0.001 \\
\hline
\end{tabular}

HR, hazard ratio; CI, confidence interval; AFP, $\alpha$-fetoprotein; TNM, tumor-node-metastasis; ELOVL6, elongation of very long-chain fatty acids family member 6 .

Table III. Univariate and multivariate analyses of hepatocellular carcinoma patient variables for disease-free survival.

\begin{tabular}{lccccc}
\hline & \multicolumn{3}{c}{ Univariate analysis } & & \multicolumn{2}{c}{ Multivariate analysis } \\
\cline { 2 - 4 } Variable & HR & $95 \% \mathrm{CI}$ & P-value & HR & 95\% CI \\
\hline Age, years & 0.982 & $0.969-0.995$ & 0.005 & & \\
Sex & 0.805 & $0.510-1.271$ & 0.352 & & \\
AFP & 1.244 & $0.852-1.817$ & 0.258 & & \\
Cirrhosis & 1.066 & $0.707-1.607$ & 0.760 & & $1.089-1.998$ \\
Tumor size, cm & 1.406 & $1.001-1.975$ & 0.050 & & $1.062-2.058$ \\
Differentiation & 1.626 & $0.924-2.862$ & 0.411 & & 0.032 \\
TNM stage & 1.442 & $1.065-1.952$ & 0.018 & & 0.041 \\
Vascular invasion & 1.954 & $1.332-2.868$ & 0.001 & & 1.475 \\
ELOVL6 expression & 1.441 & $1.036-2.004$ & 0.030 & & 1.478 \\
\hline
\end{tabular}

HR, hazard ratio; CI, confidence interval; AFP, $\alpha$-fetoprotein; TNM, tumor-node-metastasis; ELOVL6, elongation of very long-chain fatty acids family member 6 .
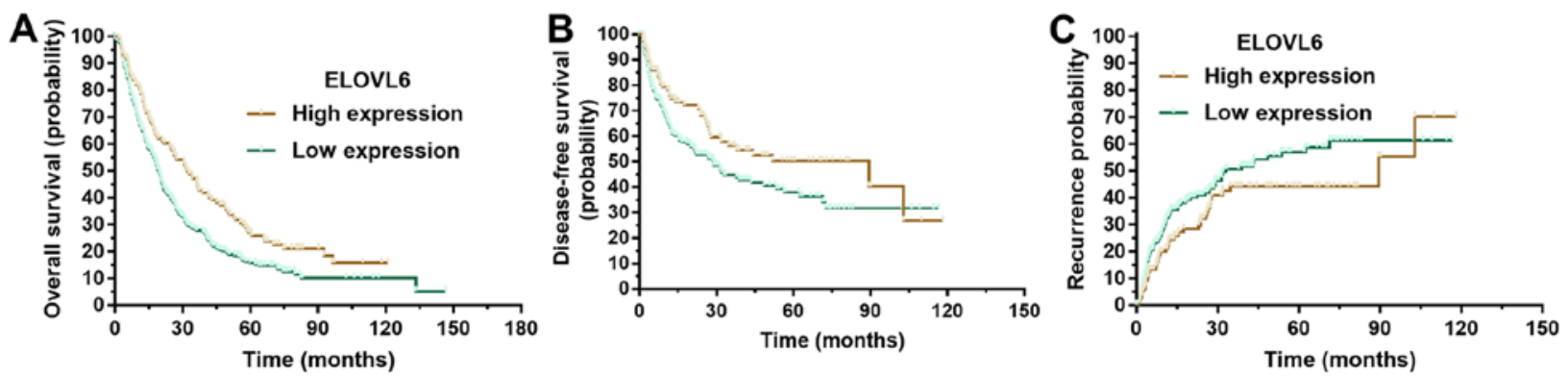

Figure 2. Prognostic values for ELOVL6 expression level in patients with HCC. Kaplan-Meier analysis revealed that (A) patients with low expression levels of ELOVL6 had significantly poorer overall survival times $(\mathrm{P}<0.001)$. Compared with the patients with high ELOVL6 expression levels, patients with low ELOVL6 expression had significantly (B) lower disease-free survival times $(\mathrm{P}=0.029)$ and $(\mathrm{C})$ a higher probability of recurrence $(\mathrm{P}=0.044)$. ELOVL6, elongation of very long-chain fatty acids family member 6; HCC, hepatocellular carcinoma.

Previous studies have revealed that the level of palmitic and stearic acid in tumor cells was associated with the prognosis of cancer patients $(39,40)$. Bougnoux et al (41) found that breast cancer patients with high levels of stearic acid in their tumors had a lower likelihood of these tumors metastasizing. Further studies also revealed that patients with breast cancer 
Table IV. Univariate and multivariate analyses of hepatocellular carcinoma patient variables for recurrence.

\begin{tabular}{|c|c|c|c|c|c|c|}
\hline \multirow[b]{2}{*}{ Variable } & \multicolumn{3}{|c|}{ Univariate analysis } & \multicolumn{3}{|c|}{ Multivariate analysis } \\
\hline & HR & $95 \% \mathrm{CI}$ & P-value & HR & $95 \% \mathrm{CI}$ & P-value \\
\hline Age, years & 0.836 & $0.612-1.143$ & 0.262 & & & \\
\hline Sex & 0.817 & $0.505-1.320$ & 0.408 & & & \\
\hline AFP & 1.122 & $0.760-1.654$ & 0.563 & & & \\
\hline Cirrhosis & 1.067 & $0.690-1.648$ & 0.771 & & & \\
\hline Tumor size, $\mathrm{cm}$ & 1.162 & $0.823-1.641$ & 0.392 & & & \\
\hline Differentiation & 1.432 & $0.811-2.528$ & 0.215 & & & \\
\hline TNM stage & 1.378 & $1.002-1.897$ & 0.049 & & & \\
\hline Vascular invasion & 1.839 & $1.217-2.778$ & 0.004 & 1.773 & $1.196-2.674$ & 0.019 \\
\hline ELOVL6 expression & 1.298 & $1.122-1.827$ & 0.030 & 1.421 & $1.156-1.983$ & 0.045 \\
\hline
\end{tabular}

HR, hazard ratio; CI, confidence interval; AFP, $\alpha$-fetoprotein; TNM, tumor-node-metastasis; ELOVL6, elongation of very long-chain fatty acids family member 6 .
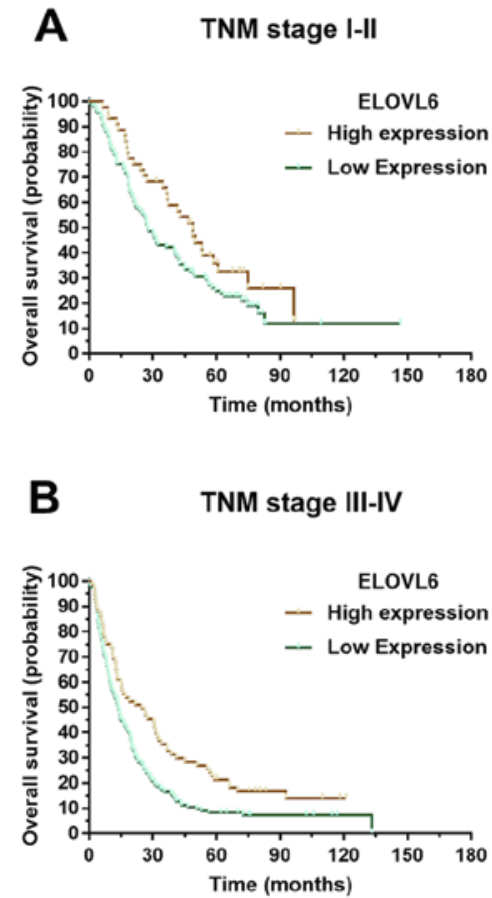
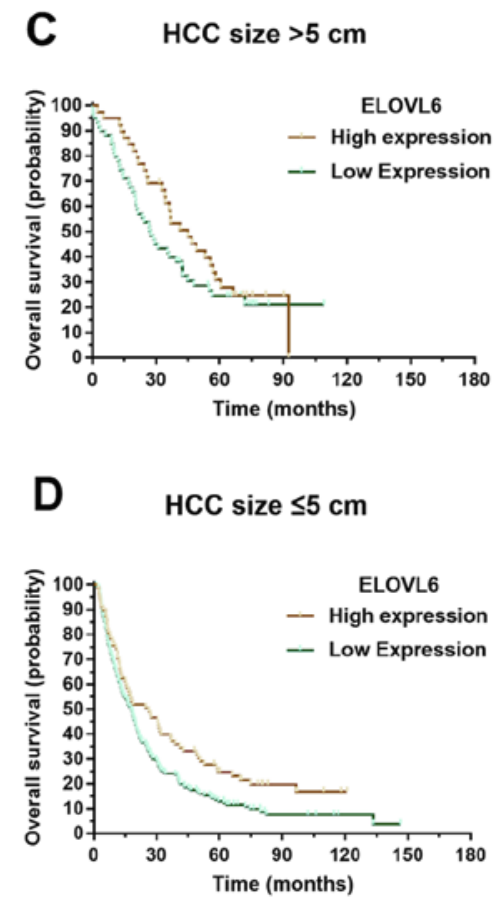
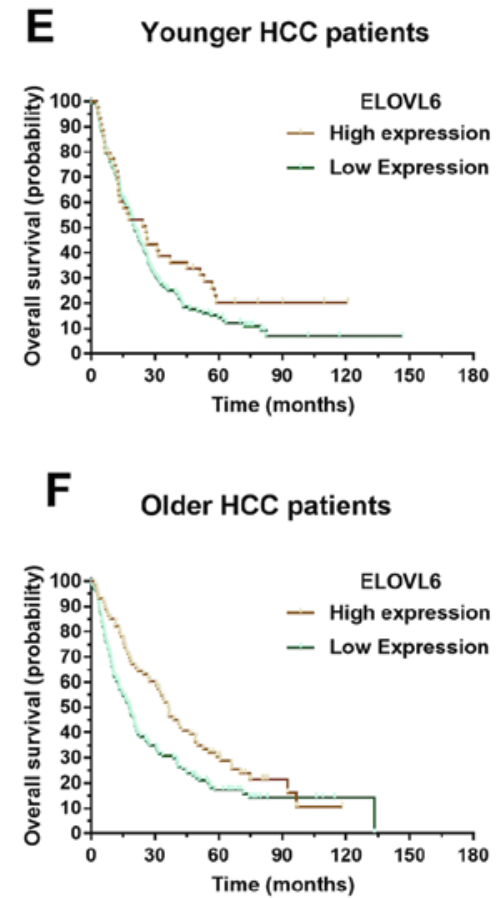

Figure 3. Prognostic prediction values of ELOVL6 expression level in subgroup analysis of patients with HCC. The overall survival between patients with high and low expression levels of ELOVL6: (A) TNM stage I-II HCCs, P=0.048; (B) stage III-IV HCCs, P=0.003. (C) In HCC size >5 cm HCCs, P=0.014; (D) $\leq 5 \mathrm{~cm} \mathrm{HCCs}, \mathrm{P}=0.042$. (E) Younger patients with HCC, $\mathrm{P}=0.043$; and (F) older patients with HCC, $\mathrm{P}=0.014$. ELOVL6, elongation of very long-chain fatty acids family member 6; HCC, hepatocellular carcinoma.

and elevated palmitic acid levels had a poorer prognosis, and that the expression of the ELOVL6 gene was significantly downregulated in these patients (42-44). In the present study, the overall and disease-free survival time of patients with high ELOVL6 expression levels was increased. However, the hypothesis that ELOVL6 regulates intracellular lipid components and influences the prognosis of patients requires further confirmation. Lipid metabolism is a key aspect of tumor growth; fatty acids not only serve as an energy source for tumors, but as a cellular component of rapidly proliferating tumor cells. ELOVL6 extends the carbon chain of fatty acids and inhibits their use, which may be detrimental in rapid tumor proliferation. Additional studies have confirmed that ELOVL6 is involved in both migration and proliferation $(45,46)$, and in the present study, ELOVL6 expression was associated with vascular invasion, which is also closely associated with factors such as vascular endothelial growth factor. The majority of chronic liver diseases are associated with hypoxic symptoms that come with with metabolic diseases, such as non-alcoholic fatty liver disease. Chronic hypoxia can result in the disorder of lipid metabolism and an increase in vascular endothelial growth factor expression in hepatocytes, thereby increasing 
blood flow in the liver to adapt to the anoxic environment. In $\mathrm{HCC}$, the formation of these microvessels also increases the migration ability of tumor cells $(47,48)$. Vascular invasion of tumors is a complex process that utilizes the motility of tumor cells and the proliferation and migration of vascular endothelial cells $(49,50)$. The molecular mechanism of vascular invasion is not fully explained by ELVOL6 expression; this may explain why vascular invasion was associated with ELOVL6 expression in the present study, but that they were also independent prognostic factors. It was demonstrated that the lower the expression level of ELOVL6, the higher the probability of vascular invasion, which may be due to the decreased expression level of ELOVL6 and subsequent increase in tumor cell migration. However, this theory requires further experimental confirmation.

Although ELOVL6 is involved in lipid synthesis (46), in order to meet the requirements of rapidly proliferating tumor cells, over-activated lipid-synthesized fatty acids are used to synthesize cell membranes and other organelles, rather than being stored in lipid droplets $(11,51)$. In other tumor types, the expression of ELOVL6 was also found to be decreased (52), but in order to confirm the role of ELOVL6 in HCC, further in vivo and in vitro experimentation is required.

There are some limitations to the present study; the sample size was relatively small, which may have introduced a degree of bias. The data were also collected from a single institution, which may also have resulted in enrollment bias. A multicenter prospective study is warranted to further validate the role and potential prognostic value of ELOVL6 in HCC.

In summary, the present study highlighted a role for ELOVL6 in the development and progression of HCC. The data revealed that ELOVL6 expression level was decreased in HCC tissues, which was significantly correlated with tumor size. High ELOVL6 expression level correlated with longer survival times in patients with HCC, and therefore, ELOVL6 may serve as an independent factor for improved patient outcome. Collectively, the present study suggested that ELOVL6 may be a promising biomarker for the prognosis of patients with HCC, and a potential target for HCC treatment.

\section{Acknowledgements}

Not applicable.

\section{Funding}

No funding was received.

\section{Availability of data and materials}

The datasets used and/or analyzed during the current study are available from the corresponding author on reasonable request.

\section{Authors' contributions}

MD designed the study. HL and MD wrote the manuscript. XW and JT analyzed and interpreted the patient data. HL and HZ performed the experiments. All authors read and approved the final manuscript.

\section{Ethics approval and consent to participate}

The present study was approved by the Medical Ethics Committee of Jining No. 1 People's Hospital. All procedures followed were in accordance with the ethical standards of the responsible committee on human experimentation and with the Helsinki Declaration 2008.

\section{Patient consent for publication}

As a retrospective study, the Medical Ethics Committee waived the need for informed patient consent.

\section{Competing interests}

The authors declare that they have no competing interest.

\section{References}

1. Clavien PA, Lesurtel M, Bossuyt PM, Gores GJ, Langer B and Perrier A; OLT for HCC Consensus Group: Recommendations for liver transplantation for hepatocellular carcinoma: An international consensus conference report. Lancet Oncol 13: e11-e22, 2012.

2. Poon D, Anderson BO, Chen LT, Tanaka K, Lau WY, Van Cutsem E, Singh H, Chow WC, Ooi LL, Chow P, et al: Management of hepatocellular carcinoma in Asia: Consensus statement from the Asian Oncology Summit 2009. Lancet Oncol 10: 1111-1118, 2009.

3. Cai SH, Lu SX, Liu LL, Zhang CZ and Yun JP: Increased expression of hepatocyte nuclear factor 4 alpha transcribed by promoter 2 indicates a poor prognosis in hepatocellular carcinoma. Therap Adv Gastroenterol 10: 761-771, 2017.

4. Ou H, Cai S, Liu Y, Xia M and Peng J: A noninvasive diagnostic model to assess nonalcoholic hepatic steatosis in patients with chronic hepatitis B. Therap Adv Gastroenterol 10: 207-217, 2017.

5. Forner A, Llovet JM and Bruix J: Hepatocellular carcinoma. Lancet 379: 1245-1255, 2012.

6. Friemel J, Rechsteiner M, Frick L, Böhm F, Struckmann K, Egger M, Moch H, Heikenwalder M and Weber A: Intratumor heterogeneity in hepatocellular carcinoma. Clin Cancer Res 21: 1951-1961, 2015.

7. Wu X, Cai S, Li Z, Zheng C, Xue X, Zeng J and Peng J: Potential effects of telbivudine and entecavir on renal function: A systematic review and meta-analysis. Virol J 13: 64, 2016.

8. Cai S, Ou Z, Liu D, Liu L, Liu Y, Wu X, Yu T and Peng J: Risk factors associated with liver steatosis and fibrosis in chronic hepatitis B patient with component of metabolic syndrome. United European Gastroenterol J 6: 558-566, 2018.

9. Zeng J, Cai S, Liu J, Xue X, Wu X and Zheng C: Dynamic changes in liver stiffness measured by transient elastography predict clinical outcomes among patients with chronic Hepatitis B. J Ultrasound Med 36: 261-268, 2017.

10. CD36-mediated lipid metabolism promotes metastasis. Cancer Discov 7: F12, 2017.

11. Xiao YB, Cai SH, Liu LL, Yang X and Yun JP: Decreased expression of peroxisome proliferator-activated receptor alpha indicates unfavorable outcomes in hepatocellular carcinoma. Cancer Manag Res 10: 1781-1789, 2018.

12. Li Z and Kang Y: Lipid metabolism fuels cancer's spread. Cell Metab 25: 228-230, 2017.

13. Nieman KM, Kenny HA, Penicka CV, Ladanyi A, Buell-Gutbrod R, Zillhardt MR, Romero IL, Carey MS, Mills GB, Hotamisligil GS, et al: Adipocytes promote ovarian cancer metastasis and provide energy for rapid tumor growth. Nat Med 17: 1498-1503, 2011.

14. Pascual G, Avgustinova A, Mejetta S, Martín M, Castellanos A, Attolini CS, Berenguer A, Prats N, Toll A, Hueto JA, et al: Targeting metastasis-initiating cells through the fatty acid receptor CD36. Nature 541: 41-45, 2017.

15. Sunami Y, Rebelo A and Kleeff J: Lipid metabolism and lipid droplets in pancreatic cancer and stellate cells. Cancers (Basel) 10: pii: E3, 2017. 
16. Gupta S, Santra L, Naskar S, Maurya SK, Rana M, Ghosh J and Dhara SK: Heterologous expression of porcine elongase 6 (ELOVL6) gene in a human cell line. Indian J Med Res 145: 563-568, 2017.

17. Su YC, Feng YH, Wu HT, Huang YS, Tung CL, Wu P, Chang CJ, Shiau AL and Wu CL: Elovl6 is a negative clinical predictor for liver cancer and knockdown of Elovl6 reduces murine liver cancer progression. Sci Rep 8: 6586, 2018.

18. Feng YH, Chen WY, Kuo YH, Tung CL, Tsao CJ, Shiau AL and Wu CL: Elovl6 is a poor prognostic predictor in breast cancer. Oncol Lett 12: 207-212, 2016.

19. Yamashita Y, Nishiumi S, Kono S, Takao S, Azuma T and Yoshida M: Differences in elongation of very long chain fatty acids and fatty acid metabolism between triple-negative and hormone receptor-positive breast cancer. BMC Cancer 17: 589, 2017.

20. Bosquet A, Guaita-Esteruelas S, Saavedra P, Rodríguez-Calvo R, Heras M, Girona J and Masana L: Exogenous FABP4 induces endoplasmic reticulum stress in HepG2 liver cells. Atherosclerosis 249: 191-199, 2016.

21. Horie Y, Suzuki A, Kataoka E, Sasaki T, Hamada K, Sasaki J, Mizuno K, Hasegawa G, Kishimoto H, Iizuka M, et al: Hepatocyte-specific Pten deficiency results in steatohepatitis and hepatocellular carcinomas. J Clin Invest 113: 1774-1783, 2004.

22. Cai SH, Lv FF, Zhang YH, Jiang YG and Peng J: Dynamic comparison between Daan real-time PCR and Cobas TaqMan for quantification of HBV DNA levels in patients with CHB. BMC Infect Dis 14: 85, 2014.

23. Cai S, Cao J, Yu T, Xia M and Peng J: Effectiveness of entecavir or telbivudine therapy in patients with chronic hepatitis B virus infection pre-treated with interferon compared with de novo therapy with entecavir and telbivudine. Medicine (Baltimore) 96: e7021, 2017.

24. Chen WX, Cheng L, Xu LY, Qian Q and Zhu YL: Bioinformatics analysis of prognostic value of TRIM13 gene in breast cancer. Biosci Rep 39: pii: BSR20190285, 2019.

25. Li C, Zhou D, Jiang X, Liu M, Tang H and Mei Z: Identifying hepatocellular carcinoma-related hub genes by bioinformatics analysis and CYP2C8 is a potential prognostic biomarker. Gene 698: 9-18, 2019.

26. Tsochatzis EA, Meyer T and Burroughs AK: Hepatocellular carcinoma. N Engl J Med 366: 92-93, 2012.

27. White DL, Thrift AP, Kanwal F, Davila J and El-Serag HB Incidence of hepatocellular carcinoma in All 50 United States, From 2000 Through 2012. Gastroenterology 152: 812-820.e5, 2017.

28. Cai S, Li Z, Yu T, Xia M and Peng J: Serum hepatitis B core antibody levels predict $\mathrm{HBeAg}$ seroconversion in chronic hepatitis B patients with high viral load treated with nucleos(t)ide analogs. Infect Drug Resist 11: 469-477, 2018.

29. Cai S, Yu T, Jiang Y, Zhang Y, Lv F and Peng J: Comparison of entecavir monotherapy and de novo lamivudine and adefovir combination therapy in HBeAg-positive chronic hepatitis B with high viral load: 48-week result. Clin Exp Med 16: 429-436, 2016.

30. Calvisi DF, Wang C, Ho C, Ladu S, Lee SA, Mattu S, Destefanis G, Delogu S,Zimmermann A, Ericsson J, et al: Increased lipogenesis, induced by AKT-mTORC1-RPS6 signaling, promotes development of human hepatocellular carcinoma. Gastroenterology 140 1071-1083, 2011

31. Cao D, Song X, Che L, Li X, Pilo MG, Vidili G, Porcu A, Solinas A, Cigliano A, Pes GM, et al: Both de novo synthetized and exogenous fatty acids support the growth of hepatocellular carcinoma cells. Liver Int 37: 80-89, 2017.

32. Caro P, Kishan AU, Norberg E, Stanley IA, Chapuy B, Ficarro SB, Polak K, Tondera D, Gounarides J, Yin H, et al: Metabolic signatures uncover distinct targets in molecular subsets of diffuse large B cell lymphoma. Cancer Cell 22: 547-560, 2012.

33. Al-Bahlani S, Al-Lawati H, Al-Adawi M, Al-Abri N, Al-Dhahli B and Al-Adawi K: Fatty acid synthase regulates the chemosensitivity of breast cancer cells to cisplatin-induced apoptosis. Apoptosis 22: 865-876, 2017.

34. Sczaniecka AK, Brasky TM, Lampe JW, Patterson RE and White E: Dietary intake of specific fatty acids and breast cancer risk among postmenopausal women in the VITAL cohort. Nutr Cancer 64: 1131-1142, 2012.
35. Xiao F, Wang C, Yin H, Yu J, Chen S, Fang J and Guo F: Leucine deprivation inhibits proliferation and induces apoptosis of human breast cancer cells via fatty acid synthase. Oncotarget 7 : 63679-63689, 2016.

36. Hoekstra M, van der Sluis RJ, Kuiper J and Van Berkel TJ: Nonalcoholic fatty liver disease is associated with an altered hepatocyte microRNA profile in LDL receptor knockout mice. J Nutr Biochem 23: 622-628, 2012.

37. Moon YA, Ochoa CR, Mitsche MA, Hammer RE and Horton JD: Deletion of ELOVL6 blocks the synthesis of oleic acid but does not prevent the development of fatty liver or insulin resistance. J Lipid Res 55: 2597-2605, 2014.

38. Kessler SM, Simon Y, Gemperlein K, Gianmoena K, Cadenas C, Zimmer V, Pokorny J, Barghash A, Helms V, van Rooijen N, et al: Fatty acid elongation in non-alcoholic steatohepatitis and hepatocellular carcinoma. Int J Mol Sci 15: 5762-5773, 2014.

39. Crowe FL, Allen NE, Appleby PN, Overvad K, Aardestrup IV, Johnsen NF, Tjønneland A, Linseisen J, Kaaks R, Boeing H, et al: Fatty acid composition of plasma phospholipids and risk of prostate cancer in a case-control analysis nested within the European Prospective Investigation into Cancer and Nutrition. Am J Clin Nutr 88: 1353-1363, 2008.

40. Di Gangi IM, Mazza T, Fontana A, Copetti M, Fusilli C, Ippolito A, Mattivi F, Latiano A, Andriulli A, Vrhovsek U and Pazienza V: Metabolomic profile in pancreatic cancer patients: A consensus-based approach to identify highly discriminating metabolites. Oncotarget 7: 5815-5829, 2016.

41. Bougnoux P, Hajjaji N, Ferrasson MN, Giraudeau B, Couet C and Le Floch O: Improving outcome of chemotherapy of metastatic breast cancer by docosahexaenoic acid: A phase II trial. Br J Cancer 101: 1978-1985, 2009.

42. Baek JS and Cho CW: 2 -Hydroxypropyl- $\beta$-cyclodextrin-modi fied SLN of paclitaxel for overcoming p-glycoprotein function in multidrug-resistant breast cancer cells. J Pharm Pharmacol 65: 72-78, 2013

43. Cao W, Ma Z, Rasenick MM, Yeh S and Yu J: N-3 polyunsaturated fatty acids shift estrogen signaling to inhibit human breast cancer cell growth. PLoS One 7: e52838, 2012.

44. Conceicao LL, Dias MM, Pessoa MC, Pena GD, Mendes MC, Neves CV, Hermsdorff HH, Freitas RN and Peluzio MD: Difference in fatty acids composition of breast adipose tissue in women with breast cancer and benign breast disease. Nutr Hosp 33: 1354-1360, 2016.

45. Kikuchi M, Shimada M, Matsuzaka T, Ishii K, Nakagawa Y, Takayanagi M, Yamada N and Shimano H: Crucial role of Elovl6 in chondrocyte growth and differentiation during growth plate development in mice. PLoS One 11: e159375, 2016.

46. Anelli L, Zagaria A, Coccaro N, Tota G, Impera L, Minervini CF, Pastore D, Minervini A, Casieri P, Specchia G and Albano F: A novel $\mathrm{t}(4 ; 16)(\mathrm{q} 25 ; \mathrm{q} 23.1)$ associated with EGF and ELOVL6 deregulation in acute myeloid leukemia. Gene 529: 144-147, 2013.

47. Chen Q, Wang D, Li Y, Yan S, Dang H, Yue H, Ling J, Chen F, Zhao Y, Gou L, et al: LINC00628 suppresses migration and invasion of hepatocellular carcinoma by its conserved region interacting with the promoter of VEGFA. J Cell Physiol: Feb 10, 2019 doi: 10.1002/jcp.28233 (Epub ahead of print).

48. Cheng SY, Chen NF, Lin PY, Su JH, Chen BH, Kuo HM, Sung CS, Sung PJ, Wen ZH and Chen WF: Anti-invasion and Antiangiogenic effects of Stellettin B through inhibition of the Akt/Girdin signaling pathway and VEGF in Glioblastoma cells. Cancers (Basel) 11: pii: E220, 2019.

49. Chen SH, Zhang BY, Zhou B, Zhu CZ, Sun LQ and Feng YJ: Perineural invasion of cancer: A complex crosstalk between cells and molecules in the perineural niche. Am J Cancer Res 9: 1-21, 2019.

50. Labib PL, Goodchild G and Pereira SP: Molecular pathogenesis of cholangiocarcinoma. BMC Cancer 19: 185, 2019.

51. Lu JB, Cai SH, Pan YH and Yun JP: Altered epidermal fatty acid-binding protein expression in hepatocellular carcinoma predicts unfavorable outcomes. Cancer Manag Res 10: 6275-6284, 2018.

52. Li FJ, Wang HY, Feng XL, Li PP, Shu T, Zhao XH and Li B: Expression and clinical significance of ELOVL6 gene in high-grade serous ovarian carcinoma. Zhonghua Fu Chan Ke Za Zhi 51: 192-197, 2016 (In Chinese). 